\title{
Infinite Series Representation of the Exact PEP for Space-Time Coding
}

\author{
Z. Zhang, S.W.Cheung and T.I.Yuk \\ Department of Electronic \& Electrical Engineering, University of Hong Kong, Pokfulam Road, Hong Kong \\ zhizhang@eee.hku.hk, swcheung@eee.hku.hk, tiyuk@,eee.hku.hk
}

\begin{abstract}
The exact Pair-wise Error Probabilities (PEP) formulas developed for Space-Time (S-T) coding so far either are not in the closed forms or have the closed forms but need to calculate very complicated high order derivatives, and so are not easy to use. This paper derives, for $S-T$ coding in block Rayleigh fading, a new exact PEP formula which is expressed as the summation of a series of terms to the powers of 1/Signal-toNoise-Ratio (1/SNR). The result gives researchers an easier and more convenient way for bit-error-rate (BER) evaluation and optimization of S-T coded Multiple-Input-Multiple-Output (MIMO) systems. Monte Carlo simulation results of the BER performance of a 2-by-2 S-T coded system are used to verify our results.
\end{abstract}

Index Terms- Space-Time coding, exact PEP, infinite series

\section{INTRODUCTION}

Since the work on the construction of Space-Time (S-T) codes in [1], having an accurate Pair-wise Error Probabilities (PEP) formula has been an important issue for many researchers. The exact PEP formulas developed so far either require numerical approximations [2], or involve the residue [3] that is not so easy to determine in the case of having multiple poles with high orders $[2,4]$. Some are in closed forms [5 7] but need to calculate complicated high order derivatives which sometimes become impractical. These PEP formulas are quite complex in terms of analysis and interpretation. The well-known upper-bounded PEP proposed in [1] is easy to analyze, but unfortunately such upper-bound is very loose [8]. As a result, new ways are needed to express the PEP in the exact closed form. This paper derives an expression for the exact PEP of S-T codes in the form of an infinite series summation of the powers of the inverse Signalto-Noise-Ratio (i.e. 1/SNR). The interpretation of such PEP can be easily visualized. The closed-form formula for this infinite series is given and proved and the corresponding convergence interval is identified. Simulation results of a 2by-2 S-T coded system are given to verify our findings.

\section{SYSTEM MODEL}

The S-T system considered here is modeled as:

$$
R=H X+Y
$$

where $X, R, Y$ and $H$ are the transmit, receive , noise and channel matrices respectively. The channel matrix $H$ is a $M \times N$ matrix with $N$ and $M$ being the number of transmit and receive antennas, respectively. Each of the elements $h_{m, n}$ in $H$ is the channel transfer function from the $n$-th transmit antenna to the $m$-th receive antenna. For the Rayleigh fading channels, all elements $h_{m, n}$ in $H$ are independent identically distributed (i.i.d) complex Gaussian variables with zero mean and fixed variance for the real and imaginary parts. For simplicity and without loss of generality, the fixed variance is assumed to be 0.5 and the channel is assumed to be static within a transmission interval for a block of coded symbols. In (1), $X$ is a $N \times L$ coded symbol matrix where $L$ is the number of time intervals to transmit a complete codedsymbol block. Each of the elements $x_{n, t}$ in $X$ is a coded symbol transmitted from the $n$-th transmit antenna in the $t$-th transmission interval and having average symbol energy $E_{s}$. Since the information symbols in the S-T codes are encoded and transmitted in a block-to-block basis, the matrix $X$ in (1) could be regarded as a codeword in the code-book of a given $\mathrm{S}-\mathrm{T}$ coded scheme. The received signal matrix $R$ is a $M \times L$ matrix with each of its elements $r_{m, t}$ being the element received from the $m^{\text {th }}$ antenna in the $t^{\text {th }}$ transmission interval. Transmission delay is neglected here. Additive white Gaussian noise (AWGN) in the channel is modeled by a $M \times L$ matrix $Y$ with all elements being i.i.d complex Gaussian variables with zero-mean and variance $N_{0} / 2$ for the real and imaginary parts. Assume that the channel matrix $H$ can be perfectly estimated at the receiver, the MaximumLikelihood (ML) detector determines Euclidean distance:

$$
\text { Euclidean distance }=\left\{\sum_{m=1}^{M} \sum_{t=1}^{L}\left|r_{m, t}-\sum_{n=1}^{N} h_{m, n} x_{n, t}\right|^{2}\right\}
$$

and selects the codeword $X$ with the smallest Euclidean distance as the detected codeword.

\section{Exact PEP}

\section{A. Conditional PEP}

The PEP is denoted here as $P_{e}(X \rightarrow \hat{X})$ with $X$ and $\hat{X}$ as two different code-words. With the system model described in (1) and using ML detection, the conditional PEP given a channel matrix $H$ is [1]: 


$$
P_{e}(X \rightarrow \hat{X} \mid H)=Q\left(\sqrt{d^{2}(X, \hat{X}) S N R / 2}\right)
$$

where $S N R=E_{s} / N_{0}$ and:

$$
d^{2}(X, \hat{X})=\sum_{m=1}^{M} \sum_{n=1}^{N} \lambda_{n}\left|\beta_{n, m}\right|^{2}
$$

is the modified Euclidean distance between two code-words $X$ and $\hat{X} . \beta_{n, m}$ is an i.i.d. complex Gaussian random variable with zero mean and variance 0.5 for the real and imaginary parts, and $\lambda_{n}$ is the $n^{\text {th }}$ eigenvalue of the matrix $(X-\hat{X})(X-\hat{X})^{*}$ and so is non-negative, with $*$ denoting the transpose conjugate.

The exact PEP can be obtained by finding the PDF of $d^{2}(X, \hat{X})$ and then average the conditional PEP as described in the following section.

\section{B. Exact PEP using Craig formula}

The Craig formula is an alternative form of the $\mathrm{Q}$ function [9]:

$$
\begin{aligned}
& Q(x)=\frac{1}{\sqrt{2 \pi}} \int_{x}^{\infty} e^{-\frac{t^{2}}{2}} d t \\
& =\frac{1}{\pi} \int_{0}^{\pi / 2} e^{-\frac{x^{2}}{2 \sin ^{2} \theta}} d \theta
\end{aligned}
$$

Suppose among these $N$ eigenvalues $\lambda_{n}$, there are $K$ nonzero eigenvalues : $\lambda_{1} \ldots \lambda_{K}$. Averaging the conditional PEP in (3) over each $\beta_{n, m}$ using the Craig formula in (5) yields the exact PEP [5 7]:

$$
P_{e}(X \rightarrow \hat{X})=\frac{1}{\pi} \int_{0}^{\pi / 2}\left\{\prod_{i=1}^{K}\left(\frac{\sin ^{2} \theta}{\sin ^{2} \theta+\frac{\lambda_{i} S N R}{4}}\right)^{M}\right\} d \theta
$$

The integration in (6) can be calculated out in closed form [5 7] through partial fraction expansion, which however requires calculation of complicated high order derivatives and so is difficult to analyze. In this paper, we propose a new way to derive the exact PEP given by (6).

\section{Infinite Series Representation of Exact PEP}

\section{A. Theorem 1}

The exact PEP $P_{e}(X \rightarrow \hat{X})$ for our system model can be represented by an infinite series of the inverse powers of $S N R$ :

$$
\begin{aligned}
& P_{e}(X \rightarrow \hat{X})=S N R^{-K M} \prod_{i=1}^{K} \lambda_{i}^{-M} . \\
& \sum_{p=0}^{\infty}\left\{(-1)^{p} \frac{1}{2}\left(\begin{array}{c}
2(K M+p) \\
K M+p
\end{array}\right) S N R^{-p} \sum_{\substack{j_{1} \ldots j_{K}, j_{j} \geq 0 \\
j_{1}+\ldots+j_{K}=p}}\left[\prod_{i=1}^{K} \lambda_{i}^{-j_{i}}\left(\begin{array}{c}
M+j_{i}-1 \\
M-1
\end{array}\right)\right]\right\}
\end{aligned}
$$

on the convergence interval of $S N R>\frac{4}{\lambda_{\min }}$, where $\lambda_{\min }=\min \left\{\lambda_{1} . . \lambda_{K}\right\}$. The summation before the square bracket sums up all possible combinations of $K$ non-negative integers $j_{1} . . j_{i} . . j_{K}$ with the constraint that $\sum_{i=1}^{K} j_{i}=p$.

\section{B. Proof of Theorem 1}

The proof of Theorem 1 can be described by the following 4 steps.

Step 1: (6) can be rearranged as:

$$
\begin{aligned}
& P_{e}(X \rightarrow \hat{X})=\frac{1}{\pi} \int_{0}^{\pi / 2}\left\{\prod_{i=1}^{K}\left(\frac{\sin ^{2} \theta}{\sin ^{2} \theta+\frac{\lambda_{i} S N R}{4}}\right)^{M}\right\} d \theta \\
& =\frac{1}{\pi} \prod_{i=1}^{K}\left(\frac{4}{\lambda_{i} S N R}\right)^{M} \int_{0}^{\pi / 2}\left(\sin ^{2 K M} \theta\right)\left\{\prod_{i=1}^{K}\left(\frac{1}{1+\frac{4 \sin ^{2} \theta}{\lambda_{i}} S N R^{-1}}\right)^{M}\right\} d \theta
\end{aligned}
$$

Step 2: Applying the following Maclaurin series expansion:

$$
\left(\frac{1}{1+a x}\right)^{M}=\sum_{i=0}^{\infty}(-a)^{i}\left(\begin{array}{c}
M+i-1 \\
M-1
\end{array}\right) x^{i},|a x|<1
$$

to the term $\left(\frac{1}{1+\frac{4 \sin ^{2} \theta}{\lambda_{i}} S N R^{-1}}\right)^{M}$ in (8) with $S N R^{-1}$ as the argument yields: 


$$
\begin{aligned}
& P_{e}(X \rightarrow \hat{X})=\frac{1}{\pi} \prod_{i=1}^{K}\left(\frac{4}{\lambda_{i} S N R}\right)^{M} \int_{0}^{\pi / 2}\left(\sin ^{2 K M} \theta\right) . \\
& \left\{\prod_{i=1}^{K}\left[\sum_{j_{i}=0}^{\infty}\left(\frac{1}{\left(S N R \lambda_{i}\right)^{j_{i}}}\left(-4 \sin ^{2} \theta\right)^{j_{i}}\left(\begin{array}{c}
M+j_{i}-1 \\
M-1
\end{array}\right)\right)\right]\right\} d \theta
\end{aligned}
$$

In Step2, the absolute convergence interval for each Maclaurin series is:

$$
\frac{4 \sin ^{2} \theta}{\lambda_{i} S N R}<1
$$

To guarantee (11) to be valid for all values of $\theta$ and $\lambda_{i}$, the following condition must be satisfied:

$$
S N R>\frac{4}{\lambda_{\min }}, \quad \lambda_{\min }=\min \left\{\lambda_{1} \ldots \lambda_{K}\right\}
$$

Step 3: Since condition (12) guarantees the absolute convergence of the Maclaurin series in step 2, we can apply term-by-term multiplication to these infinite power series in (10) and obtain the new series:

$$
\begin{aligned}
& P_{e}(X \rightarrow \hat{X})=\frac{1}{\pi} \prod_{i=1}^{K}\left(\frac{4}{\lambda_{i} S N R}\right)^{M} \int_{0}^{\pi / 2}\left(\sin ^{2 K M} \theta\right) . \\
& \left\{\sum_{p=0}^{\infty}\left(-4 \sin ^{2} \theta\right)^{p} S N R^{-p} \sum_{\substack{j_{1}, \ldots j_{i}, j_{K} \\
j_{i} \geq 0, \sum_{i=1} j_{i}=p}}\left[\prod_{i=1}^{K} \lambda_{i}^{-j_{i}}\left(\begin{array}{c}
M+j_{i}-1 \\
M-1
\end{array}\right)\right]\right\} d \theta
\end{aligned}
$$

Step 4: Alternating the sequence of integration and summation in (13), and using the following integration result ( $M$ is a positive integer),

$$
\int_{0}^{\pi / 2}\left(\sin ^{2} \theta\right)^{M}=\frac{\pi}{2}\left(\frac{1}{4}\right)^{M}\left(\begin{array}{c}
2 M \\
M
\end{array}\right)
$$

the final result can be obtained as:

$$
\begin{aligned}
& P_{e}(X \rightarrow \hat{X})=\prod_{i=1}^{K}\left(\lambda_{i} S N R\right)^{-M} \sum_{p=0}^{\infty}\left\{\left(\frac{-1}{S N R}\right)^{p} \frac{1}{2}\left(\begin{array}{c}
2(K M+p) \\
K M+p
\end{array}\right) .\right. \\
& \left.\sum_{\substack{j_{1}, \ldots j_{i}, j_{K} \\
j_{i} \geq 0, \sum_{i=1} j_{i}=p}}\left[\prod_{i=1}^{K} \lambda_{i}^{-j_{i}}\left(\begin{array}{c}
M+j_{i}-1 \\
M-1
\end{array}\right)\right]\right\}
\end{aligned}
$$

\section{Remarks on Theorem 1}

1. The fist item in (7) $(p=0)$ was the upper bound proposed in [10], while the first and second items in (7) $(p=0,1)$ were reported in [5]. These two cases in fact can be regarded as a special case of Theorem 1 .

2. Most expressions for the closed-form exact PEPs, such as [5 7], require separate considerations of the equal and unequal eigenvalue cases. For example, if there are 3 eigenvalues, there will be 3 possible cases, i.e., 3 identical eigenvalues, 2 identical and 1 distinct eigenvalues, and 3 distinct eigenvalues, and it needs to know which case the system is in. If there are many non-zero eigenvalues, then it becomes quite complicated. However, Theorem 1 does not have such requirement which is shown in the next section.

3. Equation (7) shows that the function is composed of $S N R$ with the highest order of $-K M$. So the diversity order is $K M$ which governs the slope of the PEP curve at high SNRs.

4. (7) is comprised of a product of two items. One of them is $S N R^{-K M} \prod_{i=1}^{K} \lambda_{i}^{-M}$, the well-known asymptotic upper bound in [1] with a scalar factor $4^{K M}$. So Theorem 1 provides another interpretation for the rank and determinant criterion [1] based on this asymptotic upper bound. However, the derivation of such bound has two steps of approximation: first is to use Chernoff bound to approximate the $Q$ function and second is to neglect "1" in "high" SNR region. But Theorem 1 is obtained by expanding the exact PEP into a series which can thus provide a precise region of convergence.

5. According to the theory of calculus, on the interval of convergence, the power series of a given function is the Maclaurin series. So applying the Maclaurin polynomials to (6) with $S N R^{-1}$ as the argument yields the exact PEP given by (7). This is illustrated by the example in section IV.

\section{Example of 2-by-2 S-T coding system}

Consider a 2-by-2 S-T coding system with 2 transmit antennas and 2 receive antenna.

\section{A. Infinite series expansion of Exact PEP using Theorem 1}

Using Theorem 1, the exact PEP for the 2-by-2 S-T codes system can be expanded as:

$$
\begin{aligned}
& P_{e}(X \rightarrow \hat{X})=35\left(\lambda_{1} \lambda_{2}\right)^{-2}(S N R)^{-4}-252\left(\lambda_{1} \lambda_{2}\right)^{-2}\left(\lambda_{1}^{-1}+\lambda_{2}^{-1}\right)(S N R)^{-5} \\
& +462\left(\lambda_{1} \lambda_{2}\right)^{-2}\left(3 \lambda_{1}^{-2}+3 \lambda_{2}^{-2}+4 \lambda_{1}^{-1} \lambda_{2}^{-1}\right)(S N R)^{-6} \\
& -3432\left(\lambda_{1} \lambda_{2}\right)^{-2}\left(2 \lambda_{1}^{-3}+2 \lambda_{2}^{-3}+3 \lambda_{1}^{-2} \lambda_{2}^{-1}+3 \lambda_{2}^{-2} \lambda_{1}^{-1}\right)(S N R)^{-7} \\
& +6435\left(\lambda_{1} \lambda_{2}\right)^{-2}\left(5 \lambda_{1}^{-4}+5 \lambda_{2}^{-4}+8 \lambda_{1}^{-3} \lambda_{2}^{-1}+8 \lambda_{2}^{-3} \lambda_{1}^{-1}+9 \lambda_{2}^{-2} \lambda_{1}^{-2}\right)(S N R)^{-8} \\
& +o\left(S N R^{-9}\right)
\end{aligned}
$$

\section{B. Closed-Form Exact PEP}

In [8], the closed-form exact PEPs for S-T coding system can be derived for the equal eigenvalues case $\left(\lambda_{1}=\lambda_{2}=\lambda\right)$ as:

$$
P_{e}(X \rightarrow \hat{X})=[0.5(1-u)]^{4} \sum_{k=0}^{3}\left[\left(\begin{array}{c}
3+k \\
k
\end{array}\right)(0.5(1+u))^{k}\right], u=\sqrt{\frac{\lambda S N R}{4+\lambda S N R}}
$$

and the unequal eigenvalues case as: 


$$
\begin{gathered}
P_{e}(X \rightarrow \hat{X})=\left(\frac{\lambda_{1}}{\lambda_{1}-\lambda_{2}}\right)^{2}\left[\frac{1}{4}\left(1-u_{1}\right)^{2}\left(u_{1}+2\right)\right]-\frac{2 \lambda_{1}^{2} \lambda_{2}}{\left(\lambda_{1}-\lambda_{2}\right)^{3}}\left[0.5\left(1-u_{1}\right)\right] \\
+\left(\frac{\lambda_{2}}{\lambda_{1}-\lambda_{2}}\right)^{2}\left[\frac{1}{4}\left(1-u_{2}\right)^{2}\left(u_{2}+2\right)\right]+\frac{2 \lambda_{2}^{2} \lambda_{1}}{\left(\lambda_{1}-\lambda_{2}\right)^{3}}\left[0.5\left(1-u_{2}\right)\right] \\
u_{1}=\sqrt{\frac{\lambda_{1} S N R}{4+\lambda_{1} S N R}}, u_{2}=\sqrt{\frac{\lambda_{2} S N R}{4+\lambda_{2} S N R}}
\end{gathered}
$$

Now it is easy to verify remarks $2 \& 5$. The Maclaurin expansion on (18) in terms of $S N R^{-1}$ is (16) and the Maclaurin expansion on (17) in terms of $S N R^{-1}$ is also (16) if we set $\lambda_{1}=\lambda_{2}=\lambda$ in (16).

\section{Simulation and Numerical results:}

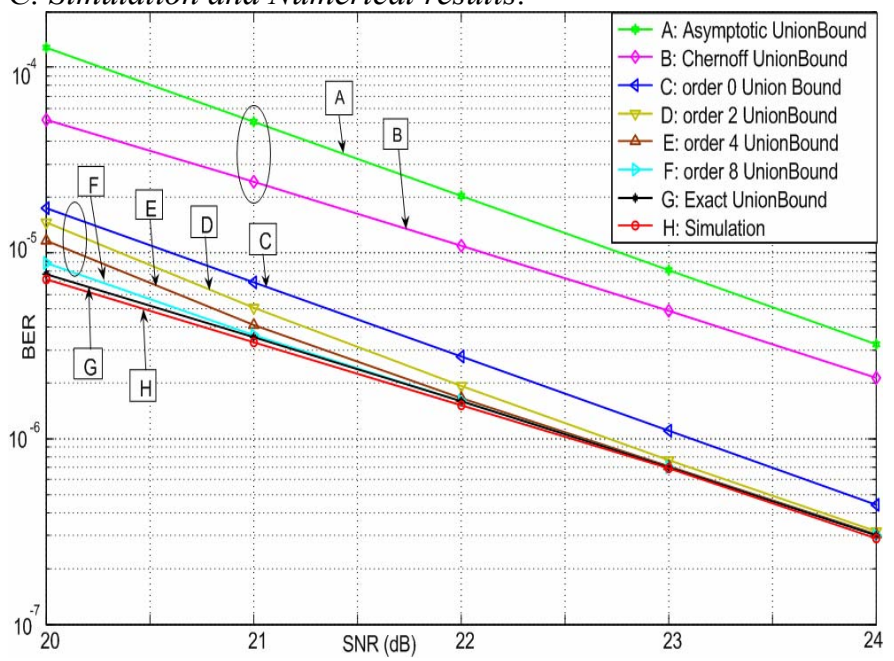

Fig 1 BER performances of $\mathrm{D}$ code using simulation and Union Bounds composed of different PEPs

Here a 2-by-2 rotation-based diagonal space-time code (D code) [11] with the optimum angle pair (1.368 rad, $0.531 \mathrm{rad})$ is used for study. It is an optimum angle pair in the sense that it leads to a minimum bit-error rate (BER) [8]. Numerical computations of the BERs using (I) the Union Bound [12] with the exact PEPs of (17) and (18), (II) the Union Bound with the Chernoff bound PEP[1], and (III) the Union Bound with the asymptotic bound PEP [1] are shown in Fig. 1 as Exact Union Bound, Chernoff Union Bound and Asymptotic Union Bound respectively. The results of using different numbers of terms in (7) to compute the Union Bound are also shown in Fig. 1. Here, order $i$, for $i=0,2,4$ and 8, refers to the PEPs obtained by using just the first $i+1$ terms in (7). Note that, in (16), only the first 5 terms of (7) are used and so it is shown as order 4 in Fig. 1. Monte Carlo simulations have also been performed and results are shown in Fig. 1 for comparison. It can be seen that the Union Bound results using the exact PEP of (17) and (18) are nearly identical to the simulation results, thus (17) and (18) are the accurate closedform exact PEPs. The Union Bounds with the Chernoff and Asymptotic bound PEPs differ from the simulation results by about $2 \sim 3 \mathrm{~dB}$ and so are loose upper bounds. However, the
Order 0 Union Bound differs from simulation by less than 1 $\mathrm{dB}$ for the SNRs tested. The difference gets smaller at higher SNRs. With Order 2, the difference is less than $0.5 \mathrm{~dB}$ at $\mathrm{SNR}=20 \mathrm{~dB}$ and is unnoticeable at SNR $>23 \mathrm{~dB}$. With Order 4, the result is the same as simulation at SNR $>21 \mathrm{~dB}$. With Order 8 , the result is identical to simulation for all the SNRs tested. Therefore the Union Bound with more terms used in (7) converges faster towards the simulation results and at lower SNR values, which verifies our proposed series representation.

\section{CONCLUSIONS}

The paper derives a new representation to the exact PEP of S$T$ code system in a block Rayleigh fading channel. The new representation is the summation of an infinite series in powers of $1 / \mathrm{SNR}$. The close-form formula of this infinite series and the corresponding convergence interval have been determined. Analytical results of the new representation on a 2-by-2 S-T code system have been verified by computer simulation results.

\section{REFERENCES}

[1]. V. Tarokh, N. Seshadri, and A. R. Calderbank, "Spacetime codes for high data rate wireless communication: Performance criterion and code construction," IEEE Trans. Inform. Theory, Mar.1998.

[2] G. Taricco and E. Biglieri, "Exact pairwise error probability of space-time codes," IEEE Trans. Inform. Theory, Feb. 2002

[3]. Uysal, M; Georghiades, C.N, “On the error performance analysis of space-time trellis codes", Wireless Communications, IEEE Trans on, July 2004,

[4] S. Benedetto and E. Biglieri, "Principles of Digital Transmission with wireless applications", Kluwer, New York, 1999, page 704

[5] H.F Lu; Y.K Wang; Kumar, P.V.; Chugg, K.M, "Remarks on space-time codes including a new lower bound and an improved code", IEEE Trans. Inform. Theory, Oct. 2003

[6] M. K. Simon and M.S. Alouini, "Digital Communication Over Fading Channels”, 2nd ed. New York: Wiley, 2005.

[7] Zinan Lin, Elza Erkip and Andrej Stefanov, "Exact Pairwise Error Probability for the MIMO Block Fading Channel", International Symposium on Information Theory and its Applications, ISITA, Italy, Oct. 2004 
[8]. Z.Zhang, S.W.Cheung, T.I.Yuk, H.Kuo, "Optimization and BER Evaluation for Space-Time codes using Union Bounds for 2-by-2 MIMO systems", IEEE 06'Spring VTC

[9]. J. W. Craig, "A new, simple and exact result for calculating the probability of error for two-dimensional signal constellations," in IEEE MILCOM '91. Boston, 1991

[10] Fitz, M.P, Grimm, J. and Siwamogsatham, S, “A new view of performance analysis techniques in correlated

Rayleigh fading", WCNC'99, IEEE, Sept 1999-

[11] H. Yao and G.Wornell, "Achieving the full MIMO diversity-multiplexing frontier with rotation-based space-time codes", Annual Allerton Conference on Communication, Control and Computing, Monticello IL, 2003

[12] Divsalar, D.; Simon, M.K., "The design of trellis coded MPSK for fading channels: performance criteria", IEEE Trans on Commu, Sept. 1988 